\title{
Optimal Traffic Grooming in WDM using Lighttours
}

\author{
Jose L. Marzo ${ }^{1} ，$ Fernando Solano $^{1} ，$ Jaudelice C. de Oliveira ${ }^{2}$ ， Luis F. Caro ${ }^{1}$ Ramon Fabregat $^{1}$ \\ ${ }^{1}$ Institute of Informatics and Applications University of Girona, Girona, Spain \\ ${ }^{2}$ Electrical and Computer Engineering Department Drexel University, Philadelphia, USA *
}

June 28, 2006

\begin{abstract}
Recent developments in optical communications have allowed simpler optical devices to improve network resource utilization. As such, we propose adding a lambda-monitoring device to a Wavelength-Routing Switch (WRS) allowing better performance when traffic is routed and groomed. This device may allow a WRS to aggregate traffic over optical routes without incurring in optical-electrical-optical conversion for the existing traffic. In other words, optical routes can be taken partially to route demands creating a sort of "lighttours". In this paper, we compare the number of OEO conversions needed to route a complete given traffic matrix using either lighttours or lightpaths.
\end{abstract}

Keywords: Lighttours, WDM, traffic grooming, OEO conversions, lambda-monitoring.

\section{INTRODUCTION}

Internet bandwidth requirements are increasing drastically nowadays and optical Wavelength-Division-Multiplexed networks (WDM) are designed to satisfy these requirements today. WDM networks usually route traffic demand by means of lightpaths. A lightpath is an end-to-end (fully) optical route between two Wavelength-Routing Switches (WRS), not necessarily neighbors. Since fibers can be demultiplexed into fewer wavelengths according to the number of traffic demands, one lightpath is used to route several low-speed demands (subwavelength demands). Therefore, low-speed demands are groomed into high-speed wavelengths channels [1], taking advantage of the high-capacity of wavelengths.

To improve resource utilization, sometimes more than one consecutive lightpath is used to forward a single demand. Therefore, a lightpath forwards several demands together and a demand is forwarded by different consecutive lightpaths. When a demand changes from one lightpath to another, packets need to be converted from optical signal to electrical data in order to decide the next lightpath to take. Once this decision has been made, packets need to be re-converted from electrical data to the optical signal so that they can be routed optically. These Optical-Electrical-Optical (OEO) conversions are usually avoided by network operators because more network resources (e.g. transmitters and receivers) are needed to regenerate optical signals. Moreover, OEO conversions could delay traffic since traffic flowing must be processed and queued electronically. Therefore, the way in which traffic is groomed affects not only the performance of the network but also its throughput.

In this paper the TRAFFIC GROOMING, ROUTING AND WAVELENGTH ASSIGNMENT (GRWA) problem (e.g., studied in [2] and [3]) is tackled. In general, the GRWA problem can be described as follows:

GIVEN: A traffic demand matrix $(\Lambda)$ and a physical optical topology including a description of its network resources: $a)$ the graph of the topology $\left.\left(G^{\prime}\left(V, E^{\prime}\right)\right), b\right)$ the number of transmitters $\left(T_{j}\right)$ and receivers $\left(R_{j}\right)$ for each node $j \in V$ and, $c)$ the wavelengths that cach link $(i, j) \in E^{\prime}$ can be demultiplexed into $(W)$,

FIND: A set of optical routes and a set of routes over these optical routes that satisfies all (or part) of the traffic demand matrix.

A novel architecture for grooming low-speed traffic demands into high-speed fully-optical routes, named Enhanced Grooming $\left(G^{+}\right)$, is proposed in this article and described in $\S 2$. The proposed architecture, $G^{+}$, allows a transparent WRS to aggregate more traffic over optical routes (lighttours) without incurring any OEO conversions for the traffic being forwarded through it. This statement is supported with a set of experiments described in $\S 3$.

Finally, in $\S 4$., conclusion regarding the proposed architecture and its model are discussed, together with proposals for future work.

${ }^{*}$ With the support of the Department of Universities, Research and Information Society (DURSI) of the Government of Catalonia and the European Social Funds (2005-SGR-00296, 2006-BE-00272 and, 2006-FIR-00109). Also with the support of the Spanish Science and Technology Ministry under contract TIC2003-05567 and, the COST project action number 293: Graphs \& Algorithms in Communication Networks. 


\section{2. $G^{+}$Network ARchitecture}

The proposed architecture $G^{+}$results of an hybrid between classical WRS architectures (named $G$ in this paper) and the RingO architecture. The former has been studied extensively and the reader is referred to [4] for a broader explanation. The later is briefly described below.

In RingO, there is only one fiber connecting all nodes. The fiber can be demultiplexed into $|W|$ wavelengths. Every node receives its traffic in the ring by dropping one fixed wavelength of the fiber; no other node is allowed to drop that particular wavelength in the ring. All nodes that want to transmit to a specific node, tune its transmitter to that particular wavelength. Therefore, RingO allows up to $|W|$ nodes in the network only.

To prevent traffic contention in the ring, RingO works under the following assumptions: $a$ ) the network transmits optical packets in fixed time-slots, $b$ ) packets are optically coded with a fixed length, $c$ ) higher layers are able to segment and reassemble optical packets and, $d$ ) the tuning times of the transmitter are smaller than the slot duration.

Furthermore, RingO nodes are equipped with a $\lambda$-monitor device that allows it to check whether packets are being transmitted over a particular wavelength $(\lambda)$ or not. The $\lambda$-monitor device is completely optical and very simple, and hence, fast. It only senses light passing through. If a $\lambda$-monitor device advices that a wavelength is free in a particular time slot for packet insertion, the RingO node may optically inject more traffic into it. Note that the existing traffic flow in this wavelength channel is not disturbed. More information of RingO can be found in $\lceil 5\rceil$.

Our proposed architecture, $G^{+}$takes advantage of the wavelength switching flexibility in $G$ for mesh networks and the way traffic is added to wavelengths (without incurring in OEO conversions for the existing traffic in the wavelength) in RingO. Basically, this is accomplished by using the RingO node architecture as a base and adding a wavelength switch fabric device just before the $\lambda$-monitor device. This architecture can be depicted in Figure 1 .

$G^{+}$architecture works under the same assumptions as in RingO; e.g, there is an optical packet network in which every packet is codified with a fixed length. A $G^{+}$WRS works as follows:

- Every single fiber is first amplified (Amp) and then, demultiplexed into a set of $W$ wavelengths.

- All the wavelengths coming from different fiber ports traverse the Wavelength Switch Fabric device. This device may either redirect wavelengths to the WRS controller or switch wavelengths fibers and/or colors.

- The wavelength going to the WRS controller (dropped wavelengths) are received by an array of receivers ("High bit-rate burst mode receiver set" in Figure 1) which converts the wavelengths to the electronic domain. This electronic traffic is given to the WRS controller for further electronic processing.

- The WRS controller can either take this traffic out of the WSR to local electronic equipments attached to low-speed ports, or queue it electronically for further optical forwarding using another wavelength. This decision is made by the WRS controller based on the electronic packet headers.

- The incoming traffic to the WRS arriving from low-speed ports is queued locally together with dropped wavelength traffic.

- The remaining wavelengths in the optical domain are tapped and a fraction of the light is redirected to the $\lambda$-monitor device.

- The $\lambda$-monitor determines whether optical packets are transiting through a wavelength or not. This is normally performed using a DC-coupled photodiode array.

- The WRS controller, based on the information the $\lambda$-monitor device gives, may inject more optical packets (queued previously in the electronic domain) into that free slot by means of a set of transmitters lasers.

- The transmitters are connected to a set of multiplexers, so that new traffic can be injected directly into an outgoing fiber using an appropriate color.

- The existing data carried in the fiber is delayed so that the WRS controller decisions are synchronized and no contention might occur in the optical domain.

As stated initially in this article, the proposed enhancement allows a transparent WRS to aggregate traffic over optical routes without incurring any OEO conversions (like RingO does) for the existing traffic in the optical route. For example, if an optical route follows the path $s \rightarrow \alpha_{0} \rightarrow \alpha_{1} \ldots \alpha_{n} \rightarrow d$-where $s$ is the starting WRS of the optical route and $d$ the destination or final WRS - then, our schemes allows WRSs $\alpha_{i}, 0 \leq i \leq n$, to add more traffic without "breaking" the optical route (if the wavelength has enough bandwidth). 

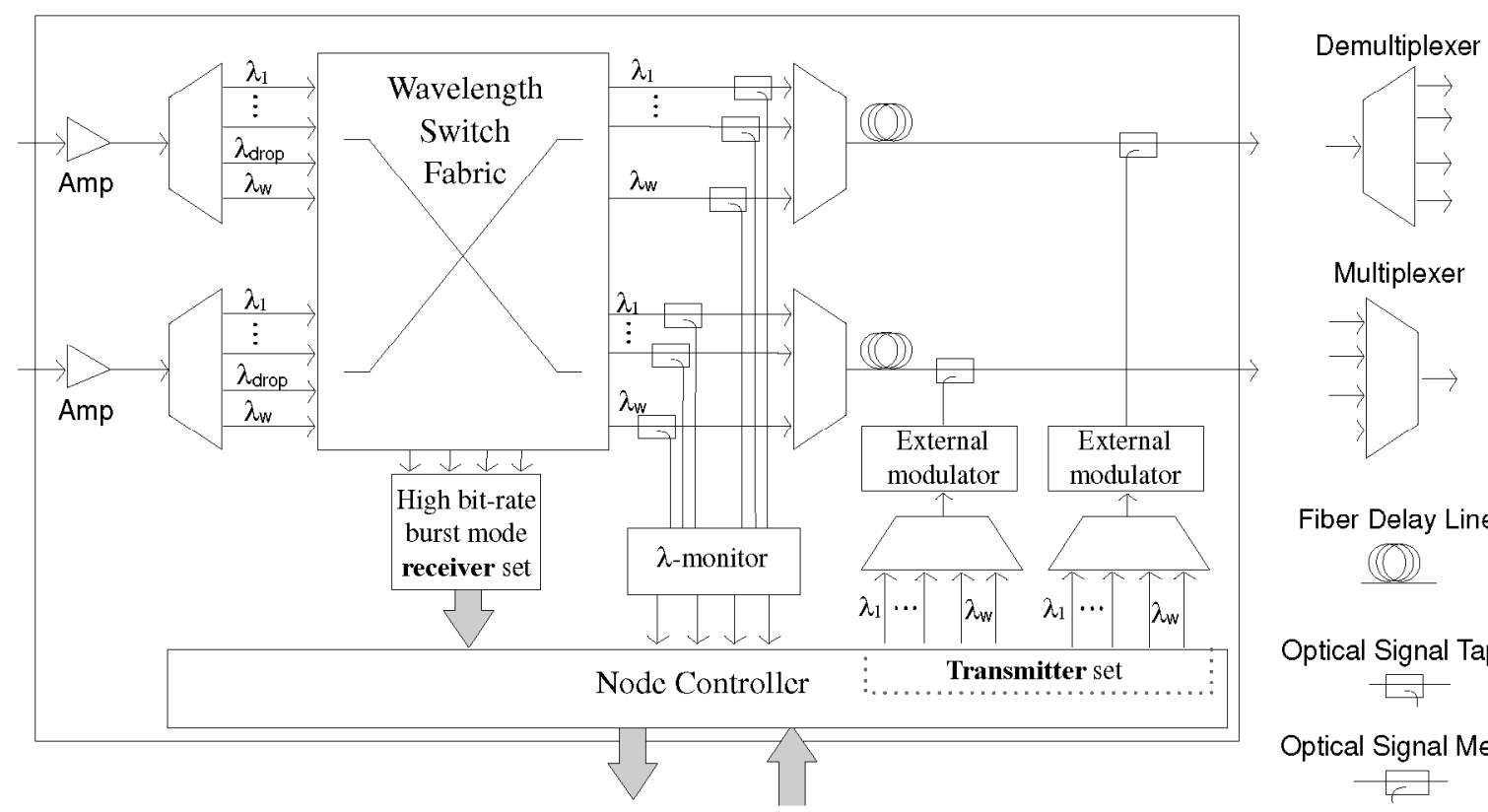

Fiber Delay Line

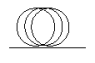

Optical Signal Tapper

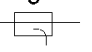

Optical Signal Merger

Traffic Output Traffic Input

Figure 1: $G^{+}$WRS architecture.

This way, the $G^{+}$"lightpaths" are able to make a tour over different traffic source WRSs and gather (en-route) their optical packets in the same optical path.

Definition 1 (LIGHTTOUR). A fully-optical path forwarding traffic from a set of WRSs to a different WRS using a $G^{+}$capable network is defined as a lighttour.

Consider the following example for analyzing $G^{+}$optical resource assignation improvement. In Figure 2, a WDM meshed network topology consisting of 4 WRSs and 5 bidirectional fiber links is shown. Fiber links can span one wavelength each. The capacity of each wavelength channel is OC-12 $2^{1}$, but all source WRSs $(s 1, s 2$ and $s 3$ ) only need a capacity of OC-3 to transmit to WRS $d$. Assume that the destination WRS $d$ has only one available receiver. For this scenario, the best solution that classical grooming may offer is to create 3 lightpaths (Figure 2(a)), one each from $s 1$ and $s 3$ to $s 2$, and another from $s 2$ to $d$. This implies that there are two OEO conversions at WRS $s 2$ for the demands coming from $s 1$ and $s 3$, therefore requiring two receivers at $s 2$. In contrast, using $G^{+}$(Figure 2(b)) the demands can be routed with a single lighttour in this topology. Using this single lighttour $(s 1 \rightarrow s 2 \rightarrow s 3 \rightarrow d$ ) the demands from $s 2$ and $s 3$ take up a part of the lighttour, i.e., they are not routed end-to-end in the lighttour. As stated, this leads to an improvement in the way the resources are used. Consequently, the network performance is also improved since less OEO conversions are needed $\left(G^{+}\right.$does not use OEO conversions at all, while $G$ solution needs 2).

Three properties that differentiate lighttours from lightpaths are discussed below.

Fact 1 (Asymmetric Bandwidth Utilization). The bandwidth utilization of a lighttour increases over the route it takes since more traffic is added along its route. On the other hand, lightpath utilization is the same in all the wavelengths it takes.

Fact 2 (Many-to-One Transmitters-Receivers Coupling). While classical grooming uses one transmitter and one receiver per lightpath, $G^{+}$may require the use of one receiver too, but possibly more than one transmitter per lighttour, emulating several consecutively-connected lightpaths at a time.

In $G^{+}$, if a lighttour uses $k$ transmitters, it is because it is adding more traffic onto the lighttour along the path (in $k-1$ intermediate transparent WRSs). With classical grooming, the same $k$ transmitters would be needed but, $k$ wavelengths and $k$ receivers might be needed as well.

Fact 3 (One-to-Many Lighttours-Virtual-Links Mapping). Since lighttours forward traffic from many WRS to a single one, a single lighttour could be seen as multiple virtual links in a virtual topology.

\footnotetext{
${ }^{1} \mathrm{OC}-1$ (optical carrier one) is equivalent to a SONET line with transmission speed of $51.84 \mathrm{Mbit} / \mathrm{s}$ using optical fiber.
} 


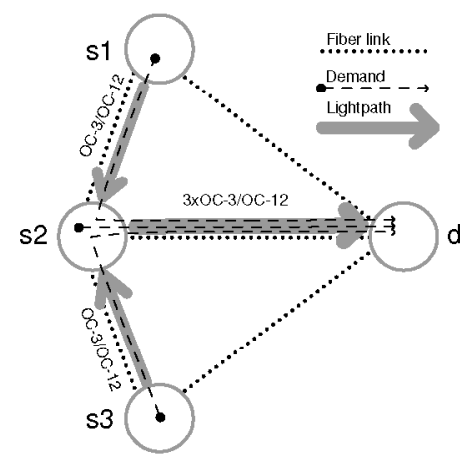

(a) G Solution.

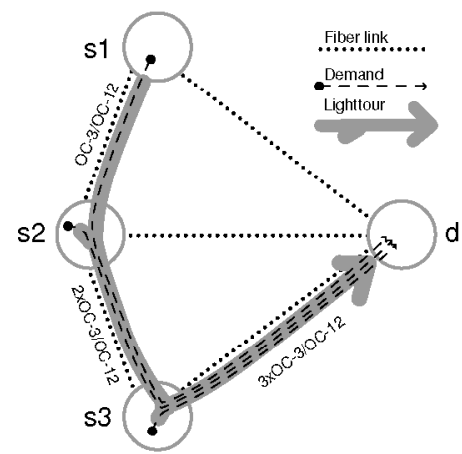

(b) $G^{+}$Solution.

Figure 2: Difference between the solutions given by classical grooming and $G^{+}$.

\section{Comparing OEO Conversions Minimization}

In this section, a set of simulations are presented with the purpose of showing the superiority of $G^{+}$. The solutions presented here are obtained by solving an ILP model which can be regarded in [6]. The network shown in Figure 3 is used in the simulations. It is considered that each fiber link may be demultiplexed to at most $|W|$ wavelengths (it may change between simulation scenarios) and that the capacity of each wavelength is OC-48. Every WRS in the network has 5 transmitters and 5 receivers.

A set of 60 randomly generated demands are considered as input to the problem?. Each demand may correspond to OC-1, OC-3 or OC-12 and is selected randomly. The selection of the source and destination WRS is done randomly a uniform distribution. The total demanded bandwidth is equivalent to OC-320.

In this subsection numerical examples minimizing the number of OEO conversions to route all traffic demands in different scenarios are presented.

The model is solved by varying two parameters: the number of wavelengths per fiber and the wavelength conversion capabilities of the WRSs. The wavelength conversion is set to full or none. When allowing full wavelength conversion, the number of wavelengths is varied from 2 to 3 . While, when wavelength conversion is not allowed, the number of wavelengths used for the simulations is varied from 3 to 4 .

The results for the cases described above are summarized in Table 1 on page 17. The columns labeled with $G^{+}$and $G$ refer to lighttours and lightpaths solutions, respectively. Each column corresponds to one solution of the model for a particular scenario.

The simulations show that $G^{+}$can route a fixed amount of traffic using half the number of $O E O$ conversions needed by classical grooming. For example, allowing full wavelength conversion at two wavelengths per fiber, $G^{+}$uses only 9 OFO conversions ( 9 demands using two virtual hops) to route all the OC-320 traffic, while classical grooming needs 19 OEO conversions ( 16 demands using two virtual hops and 1 demand using three virtual hops). As expected, the number of optical routes (and receivers in consequence) is lower, although $G^{+}$uses more transmitters.

It should be pointed out that in no solution of the lightpath-constrained model, the maximum number of re-

${ }^{2}$ The solver used is Xpress by Dash Optimization, release $2005 \mathrm{~B}$.

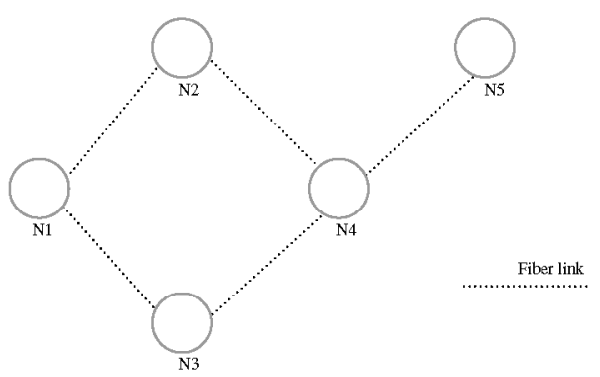

Figure 3: Physical Topology. 
Table 1: Comparison between Optimal Solutions using $G$ and $G^{+}$.

\begin{tabular}{|c|c|c|c|c|c|c|c|c|}
\hline Wv. Conv. & \multicolumn{4}{|c|}{ Yes } & \multicolumn{4}{|c|}{ No } \\
\hline \# Wavelengths & $\mid W$ & $=2$ & $\mid W$ & $=3$ & $W$ & $=3$ & $\mid W$ & $=4$ \\
\hline Grooming & $G$ & $G^{+}$ & $G$ & $G^{+}$ & $G$ & $G^{+}$ & $G$ & $G^{+}$ \\
\hline OEO Conv. & 19 & 9 & 6 & 3 & 11 & 6 & 0 & 0 \\
\hline 1-hop routing & 43 & 51 & 54 & 57 & 52 & 55 & 60 & 60 \\
\hline 2-hops routing & 16 & 9 & 6 & 3 & 5 & 4 & 0 & 0 \\
\hline$>2$-hops routing & 1 & 0 & 0 & 0 & 3 & 1 & 0 & 0 \\
\hline \# O. Routes ${ }^{1}$ & 15 & 11 & 20 & 13 & 20 & 17 & 20 & 18 \\
\hline \# Transmitters $^{2}$ & 15 & 25 & 20 & 23 & 20 & 24 & 20 & 24 \\
\hline
\end{tabular}

ceivers or transmitters is reached. Therefore, the increase in the number of OEO conversions is due to the non availability of wavelengths. Similarly, since the maximum number of transmitters is reached for the worst case pure-lighttour scenarios, we believe that a lower value of OEO conversions can be achieved by increasing the number of available transmitters in the network. However, simulating scenarios in which a fixed number of wavelengths and variable number of transmitters and receivers is used is left for further study.

On the other hand, the difference between allowing full wavelength conversions and no wavelength conversion, makes no difference in the OEO conversions, i.e., the number of OEO conversions is still reduced to half of its original value.

\section{Conclusions}

In this article we studied how a simple optical device ( $\lambda$-monitor device) considerably reduces the number of OEO conversions needed for traffic routing by enhancing grooming capabilities. We named this new way of grooming $G^{+}$.

An augmented WRS architecture is proposed including the $\lambda$-monitor device. Using this switching architecture, an improved way to create fully-optical routes is proposed. This novel routing scheme uses a new type of optical path that we call a lighttour.

To prove the advantages of $G^{+}$with respect to classical grooming, an ILP model (see [6] for details of this model) is solved in different scenarios with the objective of reducing the number of OEO conversions to route a given traffic demand matrix. The optimized solutions show a $50 \%$ reduction in the number of OEO conversions. Clearly, the number of OEO conversions is linked to the number of demands being satisfied through single-hop routing.

Since the GRWA problem has been studied widely in the last few years, we believe that focusing on the advantages that lighttours can offer in different scenarios would be interesting for the network research community.

Scenarios that we plan to tackle in further publications are automatic bandwidth provisioning, dynamic reconfiguration of lighttours, restoration or protection, multipath routing and multipath backup.

\section{REFERENCES}

[1] E. Modiano and P. J. Lin, "Traffic grouming in wdm networks," IEEE Commun. Mag., vol. 39, no. 7, pp. 124-129, July 2001.

[2] K. Zhu and B. Mukherjee, "Traffic grooming in an optical wdm mesh network," IEEE J. Select. Areas Commun., vol. 20, no. 1, pp. 122-133, Jan. 2002.

[3] J. Hu and B. Leida, "Traffic grooming, routing, and wavelength assignment in optical wdm mesh networks," in Proc. IEEE Infocom 2004, Mar. 2004, pp. 501-507.

[4] H. Z. Keyao Zhu and B. Mukherjee, "A comprehensive study on next-generation optical grooming switches," IEEE J. Select. Areas Commun., vol. 21, no. 7, pp. 1173-1186, Sept. 2003.

[5] A. Carena, V. D. Feo, J. Finochietto, R. Gaudino, F. Neri, C. Piglione, and P. Poggiolini, "Ringo: An experimental wdm optical packet network for metro applications," IEEE J. Select. Areas Commun., vol. 22, no. 8, pp. 1561-1571, Oct. 2004.

[6] F. Solano, "A xpress formulation of an ilp model for the $g^{+}$multilayer problem." Institute of Informatics and Applications, Univeristy of Girona, Tech. Rep. IIiA 06-04-RR, 2006, http://eia.udg.es/fsolanod/gplus/multilayer.mos. 